\title{
La Première Guerre mondiale au Cameroun : une guerre des archives?
}

Der Erste Weltkrieg in Kamerun: ein Krieg der Kolonialakten?

The First World War in Cameroon: a war of colonial documents?

\section{Isabell Scheele}

\section{OpenEdition}

\section{Journals}

Édition électronique

URL : https://journals.openedition.org/ceg/2154

DOI : $10.4000 /$ ceg. 2154

ISSN : 2605-8359

\section{Éditeur}

Presses Universitaires de Provence

\section{Édition imprimée}

Date de publication : 15 juin 2014

Pagination : 229-242

ISSN : 0751-4239

Référence électronique

Isabell Scheele, «La Première Guerre mondiale au Cameroun : une guerre des archives ? ", Cahiers

d'Études Germaniques [En ligne], 66 | 2014, mis en ligne le 17 décembre 2017, consulté le 28 juin 2022 URL : http://journals.openedition.org/ceg/2154; DOI : https://doi.org/10.4000/ceg.2154 


\title{
La Première Guerre mondiale au Cameroun : une guerre des archives?
}

\author{
Isabell SCHEELE \\ Université d'Aix-Marseille / Universität Tübingen
}

« La culture est l'âme d'un peuple, les archives en sont la mémoire ${ }^{1}$ ». Cette citation d'Alain Moreau souligne l'importance primordiale des archives qui conservent les traces passées d'un peuple, de son histoire et de sa culture, de ce que Moreau appelle son "âme », c'est-à-dire son être profond, sa pensée, son évolution et son identité. Elles sont l'outil qui permet la transmission du patrimoine culturel et de l'histoire aux nouvelles générations, longtemps après la disparition des témoins. C'est pourquoi elles sont l'une des principales sources de ceux qui s'appliquent à retracer l'histoire et la culture d'un peuple. Elles intéressent principalement les historiens, mais peuvent aussi servir tout autre chercheur. Outre cet aspect culturel et historique, le juriste Thomas Fitchen ${ }^{2}$ indique deux autres fonctions que l'on pourrait qualifier d'administrative et de juridique. Selon lui, les archives garantiraient les droits des citoyens, auraient valeur de preuve de leurs droits en cas de réclamation ou de succession. D'autre part, elles seraient indispensables à l'exercice du pouvoir et assureraient la continuité légale de l'administration, qui pourrait mettre à disposition ces informations ou y recourir en cas d'hésitation sur la démarche à suivre. Ainsi, toute administration ou institution dispose d'archives, qui se composent en général de règlements, de notes, de rapports, de correspondances ainsi que de

\footnotetext{
1 Alain MOREAU, cité d'après Archives nationales d'Outre-Mer (ANOM), http://www.archivesnationales.culture.gouv.fr/ anom/fr/Presentation/Fonds.html, consulté le 04.12.2013. Les Archives nationales n'ont pas pu indiquer à l'auteure la référence exacte de cette citation.

2 Thomas FitsCheN, « Das rechtliche Schicksal von staatlichen Akten und Archiven bei einem Wechsel der Herrschaft über Staatsgebiete », Saarbrücker Studien zum internationalen Recht, Baden-Baden, Nomos, 2004, p. 21, 27 et 33; voir aussi : Friedrich BENNINGHOVEN, " Verbleib, Vernichtung und Ersatz Reichs- und preußischer Behördenüberlieferungen », Der Archivar, ${ }^{\circ} 31,1978$, p. 35-38 ; Heinz BOBERACH, « Die schriftliche Überlieferung der Behörden des Deutschen Reiches 1871-1945. Sicherung, Rückführung, Ersatzdokumentation », in Heinz BoBerach, Hans Booms (dir.), Aus der Arbeit des Bundesarchivs. Beiträge zum Archivwesen, zur Quellenkunde und Zeitgeschichte, Boppard a. Rh., Boldt, 1977.
} 
documents iconographiques ou filmiques. Leur particularité est d'être constituées d'exemplaires uniques (sauf pour les duplicata) et originels. Rappelons par ailleurs la distinction faite entre les archives courantes, qui sont toujours en cours d'utilisation, et les archives historiques, lesquelles ne sont plus employées régulièrement par l'administration, mais archivées et souvent ouvertes à la recherche scientifique.

Elles constituent un témoignage irremplaçable sur le passé d'un peuple ; c'est pourquoi leurs destructions récurrentes au cours des guerres posent une série de problèmes qu'il s'agit de déterminer ici. Nous nous intéresserons dans le présent article au cas de la disparition d'archives pendant la Première Guerre mondiale, lors de l'affrontement entre les troupes allemandes et les forces alliées dans le protectorat allemand du Cameroun. Pour éviter que leur documentation ne tombe entre les mains des Alliés, l'administration allemande en a alors détruit une partie ${ }^{3}$. Il s'agissait avant tout de ne pas laisser à l'ennemi des documents pouvant contenir des informations stratégiques pour des opérations militaires, telles que les correspondances secrètes et les cartes. En effet, en tant que source d'information, les archives seraient aussi une "clé du pouvoir », pour reprendre le terme de Prakash Menon ${ }^{4}$.

Nous tenterons donc de répondre à la question suivante : dans quelle mesure la Première Guerre mondiale dans la colonie du Cameroun a-t-elle aussi été une guerre des archives, pour quels motifs et avec quels effets? Il convient, dans un premier temps, de dresser un bref tableau du sort des archives allemandes pendant la guerre, en essayant de retracer si elles ont été laissées sur place ou bien emportées, voire détruites. Puis, nous verrons comment les forces françaises du Condominium ont fait face à un manque partiel de documentation sur l'administration coloniale allemande dans le territoire nouvellement conquis. Dans un dernier temps, nous concentrerons notre attention sur l'utilisation particulière que les puissances européennes ont faite des documents coloniaux : certains extraits d'archives ont, en effet, été publiés dans la presse pour attaquer l'adversaire sur un plan moral.

\section{Les archives dans la guerre : entre destruction, évacuation et appropriation (1914-1916)}

Outre le continent européen, la Première Guerre mondiale a également investi des champs de bataille secondaires, dont les colonies allemandes en

\footnotetext{
${ }^{3}$ Berlin avait dès 1902 donné l'ordre de se restreindre à une guérilla en cas de guerre ( " hinhaltenden Kleinkrieg »), d'évacuer rapidement le personnel administratif et de ne rien laisser de précieux à l'ennemi («z.B. die Gegenstände des Signal- und Nachrichtendienstes »), BArch R1001/6878, Maßnahmen für den Kriegsfall, Berlin, 17.03.1902, Der Chef des Admiralstabes an Ausw.Amt, Kol.-Abth., Ganz Geheim.

${ }^{4}$ Prakash K. Menon, The Succession of States in Respect to Treaties, State Property, Archives and Debts, Lewiston, E. Mellen Press, 1991, p. 122.
} 
Afrique, en Asie et dans les îles du Pacifique. Le rôle de ces dernières dans l'issue de la guerre mondiale a été négligeable, et la majorité d'entre elles ont été conquises très rapidement par les Alliés, ce qui ressort clairement du tableau suivant ${ }^{5}$ :

\begin{tabular}{|l|c|c|l|l|r|}
\hline Colonie & $\begin{array}{l}\text { Contingent } \\
\text { des troupes } \\
\text { allemandes }\end{array}$ & $\begin{array}{l}\text { Contingent } \\
\text { des troupes } \\
\text { alliées }\end{array}$ & $\begin{array}{l}\text { Début des } \\
\text { hostilités }\end{array}$ & $\begin{array}{l}\text { Fin des } \\
\text { hostilités / } \\
\text { Capitulation }\end{array}$ & $\begin{array}{l}\text { Durée des } \\
\text { hostilités }\end{array}$ \\
\hline $\begin{array}{l}\text { Îles du } \\
\text { Pacifique }\end{array}$ & 140 & $\begin{array}{r}\text { pas de } \\
\text { données }\end{array}$ & 11 sept. 1914 & 21 sept. 1914 & 10 jours \\
\hline Togo & 1500 & $\begin{array}{l}\text { pas de } \\
\text { données }\end{array}$ & 06 août 1914 & 27 août 1914 & $\begin{array}{r}3 \\
\text { semaines }\end{array}$ \\
\hline Kiautschou & 3.500 & 60000 & 23 août 1914 & 07 nov. 1914 & semaines \\
\hline $\begin{array}{l}\text { Sud-Ouest } \\
\text { africain } \\
\text { allemand }\end{array}$ & 6.000 & 67000 & janv. 1915 & 5 juill. 1915 & 6 mois \\
\hline Cameroun & 8.000 & 18000 & 05 août 1914 & 18 fév. 1916 & 18 mois \\
\hline $\begin{array}{l}\text { Afrique } \\
\text { orientale } \\
\text { allemande }\end{array}$ & 18.200 & 160000 & 08 août 1914 & nov. 1918 & 4 ans \\
\hline
\end{tabular}

Les colonies, enserrées entre les empires français et britannique, laissaient augurer pour le gouvernement allemand une défense très difficile. Aussi l'objectif allemand n'a-t-il pas tant consisté à vaincre l'ennemi qu'à lui résister le plus longtemps possible. C'est pourquoi la principale stratégie appliquée par les troupes allemandes s'apparentait en réalité à une tactique de guérilla, avec des attaques-surprises sur de petites stations où l'on pillait munitions, armes et ravitaillement. D'autre part, pour ralentir l'avancée des Alliés, les Allemands détruisaient souvent toutes les infrastructures : chemins de fer, ponts, puits et points d'eau, mais aussi lignes et stations

\footnotetext{
5 Tableau élaboré par l'auteure d'après Uwe SCHULTE-VARENDORFF, Krieg in Kamerun, Berlin, Ch. Links Verlag, 2011, p. 18 et p. 140-142. Cette publication est la seule étude exhaustive sur la Première Guerre mondiale au Cameroun, qui est un sujet peu connu. Voir aussi Christoph CORNELISSEN, "Europäische Kolonialherrschaft im Ersten Weltkrieg ", in Wolfgang KRUSE (dir.), Eine Welt von Feinden. Der Große Krieg 1914-1918, Frankfurt/M., Fischer Taschenbuch Verl., 1997, p. 43-54 ; Michael CROWDER, «West Africa and the 19141918 War », Bulletin de l'Institut fondamental d'Afrique Noire, vol. 30/1, 1968, p. 227-247 ; Stefanie MiCHELS, «Totale Mobilmachung in Afrika. Der Erste Weltkrieg in Kamerun und Deutsch-Ostafrika ", in Arndt BAUERKÄMPER, Elise JULIEN (dir.), Durchhalten! Krieg und Gesellschaft im Vergleich 1914-1918, Göttingen, Vandenhoeck \& Ruprecht, 2010, p. 238-259.
} 
télégraphiques. Au Cameroun, où la guerre a duré un an et demi, cette stratégie de la terre brûlée a été étendue aux archives.

Les autorités savaient qu'en cas de guerre ou d'invasion, la communication avec la lointaine capitale risquait d'être rendue rapidement impossible. C'est pourquoi toutes les informations sur le comportement à adopter en cas de guerre étaient entreposées dès 1908 dans des stations secrètes $^{6}$. Dans chaque colonie, seul un employé du service postal et télégraphique, chargé de faire des rapports, était informé de leur existence. Les gouverneurs ne devaient être informés du lieu de stockage de ces documents qu'après avoir déclaré l'état de guerre ou l'état de siège pour tout le territoire colonial. Personne d'autre ne devait avoir accès à l'information, et dans le cas d'une invasion ennemie, l'ordre imposait de détruire ces archives. Ainsi, tout a été mis en œuvre pour éviter que l'ennemi n'obtienne de précieuses informations sur les procédures militaires adverses. Toutefois, cet ordre de destruction, émis par le plus haut niveau du gouvernement militaire à Berlin, ne concernait qu'une partie des archives : celles contenant des informations protégées par le secret militaire. Les recherches demeurent infructueuses concernant l'existence d'un ordre semblable pour les documents civils. Il est probable qu'une partie non négligeable des autres archives du Cameroun, qu'elles soient administratives, juridiques, médicales ou encore ethnographiques, a été également, soit détruite, soit emportée, ce que tendent à confirmer plusieurs documents français.

Il semblerait que les archives qui n'auraient pas été détruites aient été transférées à plusieurs reprises. À partir d'un certain nombre de documents analysés $^{7}$, nous pouvons reconstituer cette trajectoire. Les archives du gouvernorat central se trouvaient dans les locaux du siège du gouverneur, à Bouéa (Cameroun). Lorsqu'en août 1914, le gouverneur major Karl Ebermaier a donné les pleins pouvoirs militaires au capitaine des troupes, Carl Zimmermann, le gouvernorat a été transféré à Douala ${ }^{8}$, siège du commandement militaire. Douala est tombée le 27 septembre 1914, mais le gouvernorat et ses archives se trouvaient déjà transférés à Yaoundé, dernier bastion de la défense allemande. Après la prise de Yaoundé le $1^{\text {er }}$ janvier $1916^{9}$, les troupes vaincues sont parvenues à traverser la frontière de la colonie voisine Río Muni, un territoire neutre espagnol. En tout, il s'agirait de près de 50000 personnes, militaires et civils allemands et africains, qui ont passé la frontière espagnole, un cas unique dans l'histoire militaire allemande ${ }^{10}$.

\footnotetext{
6 Ces stations secrètes étaient les suivantes : Gouvernement Apia, Gouvernement Herbertshöhe, Bezirksamtmannschaft Ponape, Yep u Friedrich-Wilhelms-Hafen, Landeshauptmannschaft Jaluit, Stationsleiter Saipain, BArch R1001/6878, Maßnahmen für den Kriegsfall - Allgemeines, Berlin, 25.01.1908, Der Chef des Admiralstabes an Kol. Abth.

Roger FRANCESCHI, Le mandat français au Cameroun, Paris, Sirey, 1929, FR ANOM. 2300 COL 29, Dossier 250 ; et BArch R1001/3922.

${ }^{8}$ BArch R1001/3922, Kamerun 07.08.1914, Gouverneur Ebermaier, Telegramm.

9 SCHUlte-VARENDORFF, Krieg in Kamerun, p. 29.

10 Ibid.
} 


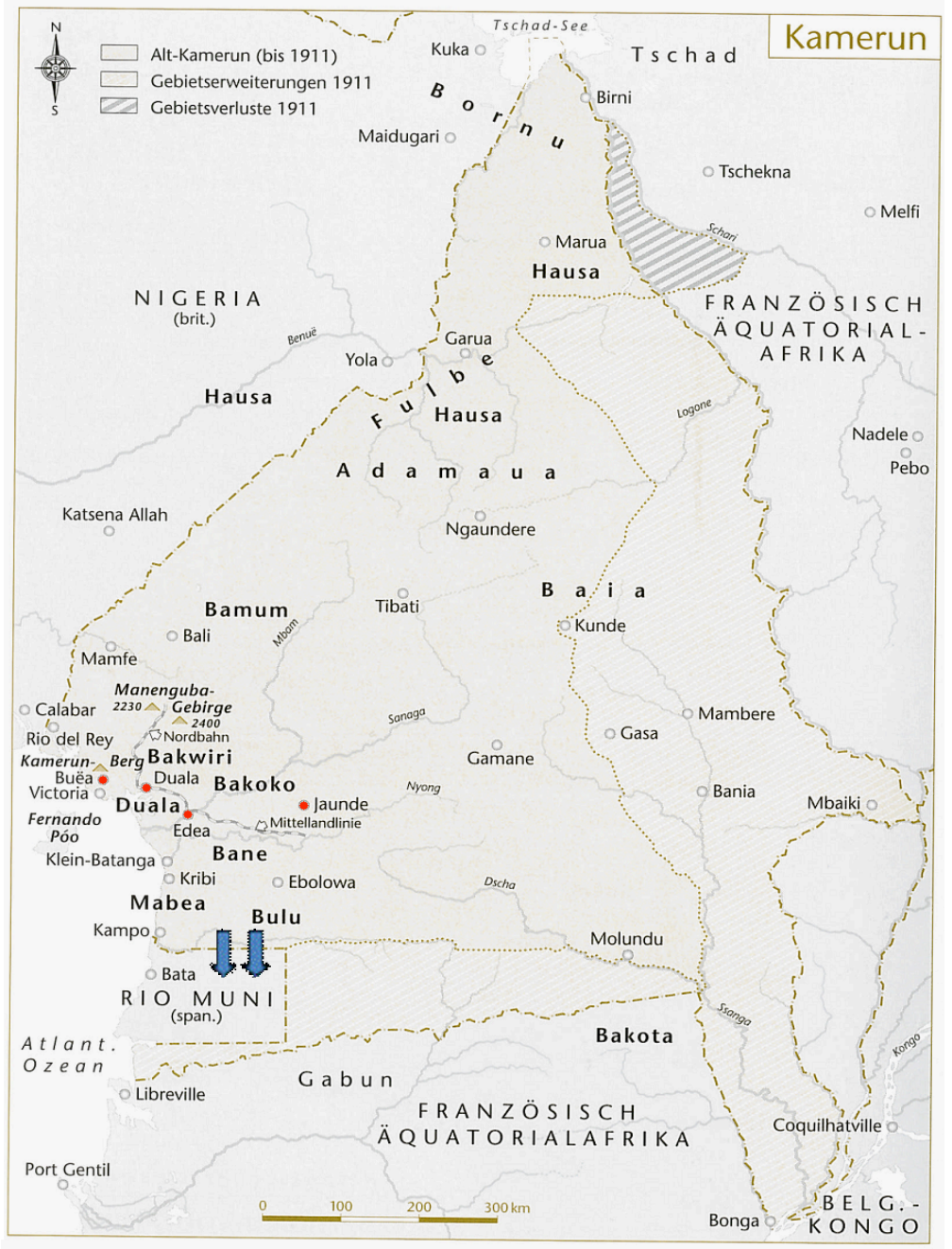

Fig. 1.- Le Cameroun en $1914^{11}$. Carte : Peter Palm, Berlin

Cette carte indique:

1. Les sièges successifs du gouvernement allemand (en rouge). Une partie des archives allemandes a probablement été déplacée entre ces différentes villes.

2. La traversée de la frontière espagnole (flèches bleues). Pour éviter d'être faits prisonniers par les Alliés, en février 1916, plus de 50000 personnes, militaires et civils allemands et africains, fuient vers le territoire voisin du Rio Muni.

11 Peter PALM, Kamerun 1914, carte extraite de Horst GRÜNDER, Gisela GRAICHEN, Deutsche Kolonien - Traum und Trauma, Berlin, Ullstein, 2005, p. 425. L'auteure a pris la liberté de modifier légèrement la carte en y ajoutant des flèches et des points de couleur. 
Le gouvernorat espagnol a permis aux civils de s'installer sur l'île Fernando Pó tandis que les soldats allemands ont été transférés dans des camps d'internement en Espagne. De là, ils ont rejoint l'Allemagne en 1918. Une large partie des archives, trop encombrantes pour être transportées, ont été laissées sur place ou brûlées, c'est du moins ce qu'affirment les sources françaises ${ }^{12}$. Il est toutefois probable qu'une petite partie ait été transportée vers Río Muni, puis, en 1919, vers l'Allemagne. Sur la carte suivante, les différentes stations citées, à savoir Bouéa, Edéa, Douala et Yaoundé sont marquées en rouge, le passage des troupes allemandes vers le territoire espagnol est indiqué par des flèches bleues.

\section{Des conflits de culture autour de la disparition des archives (1916-1918)}

Le $1^{\text {er }}$ avril 1916, les administrations française et britannique ont officiellement pris possession du territoire camerounais. Attendant l'élaboration d'un traité de paix en Europe, elles ont instauré un gouvernorat temporaire de Condominium. Les débuts de l'occupation ont été très difficiles pour les Britanniques et plus encore pour les Français ${ }^{13}$. Les quatre raisons de ces difficultés les plus couramment citées étaient : d'abord, l'absence partielle d'archives allemandes sur le fonctionnement de l'organisation administrative et économique ; puis, la disparition de la plupart des agents "indigènes", qui auraient pu instruire les nouveaux colonisateurs du fonctionnement des institutions et qui " avaient été mobilisés ou avaient suivi leurs anciens maitres ${ }^{14}$. ». Ensuite, en raison de la guerre, les activités commerciales avaient cessé, faisant stagner l'économie du pays, et les entrepreneurs allemands, ne faisant confiance ni aux agents africains ni aux Alliés, avaient fermé leurs commerces, dont ils avaient emporté la caisse et les archives :

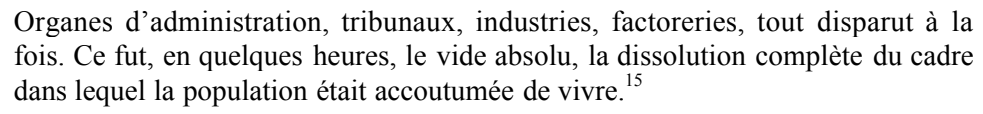

Enfin, la population africaine avait beaucoup souffert pendant une guerre qu'elle n'avait pas voulue et dont elle ne connaissait, ni les tenants, ni les aboutissants. L'administration française notait la nervosité de la population camerounaise en raison de ce qu'elle appelait les « atrocités commises par les

\footnotetext{
${ }^{12}$ FRANCESCHI, Le mandat français, p. 58, 70, 237-240 ; FR ANOM 2300 COL 29, Dossier 254 ; Martial MerLin, Brazzaville 06.10.1915, Le Gouverneur de l'AEF à Monsieur le Ministre des Colonies, Rapport, FR ANOM 2300 COL 21, Dossier 187, p. 2-9.

${ }^{13}$ MERLIN, Rapport du 06.10.1915, FR ANOM 2300 COL 21 ; voir également FRANCESCHI, « La conquête, l'occupation franco-anglaise et le Condominium. L'administration provisoire française », in FRANCESCHI, Le mandat français, p. 58-96.

${ }^{14}$ FRANCESCHI, Le mandat français, p. 58.

15 MERLIN, Rapport du 06.10.1915, FR ANOM 2300 COL 21, p. 7.
} 
Allemands ${ }^{16}$ » pendant la guerre. La situation était incertaine, il était déconseillé de sortir la nuit et de s'éloigner des localités occupées par les troupe ${ }^{17}$. La première préoccupation de la nouvelle administration a été d'assurer le calme et l'ordre dans le pays. Or, seule une administration efficace pouvait être considérée comme un vecteur de cet ordre. Les colonisateurs européens étaient en effet convaincus qu'une population africaine, sans autorité extérieure, était encline à sombrer dans l'anarchie ${ }^{18}$. Le Condominium a donc dû élaborer au plus vite, avec des moyens matériels et humains limités, une organisation nouvelle : « il a fallu aller au plus pressé, improviser ${ }^{19}$ », commentait Martial Henri Merlin, le gouverneur de l'Afrique Équatoriale Française, tentant d'organiser le gouvernorat provisoire.

Il n'est pas absurde de soutenir que ce manque de documentation ne pouvait que susciter un conflit de cultures auquel les nouveaux colonisateurs ont dû faire face : ils se sont vus face à des peuples africains largement méconnus, sur lesquels ils ne pouvaient pas s'instruire au moyen d'une documentation écrite. En l'absence des archives allemandes sur le Cameroun, les Français se sont retrouvés largement en terre inconnue. Ils ont alors dû faire une deuxième fois ce travail ethnologique sur des populations que les colons allemands s'étaient déjà efforcés de mieux connaître. Or, gérer une population rendue nerveuse, désorientée par la guerre, était une source de conflits potentiels. Et les correspondances françaises confirment que les débuts du Condominium ont été difficiles ${ }^{20}$. En privant l'administration française de l'acquisition de cette documentation ethnologique, juridique, médicale et administrative, le gouvernorat allemand du protectorat risquait donc de provoquer un conflit de cultures entre Français et Africains. A l'inverse, les Français se sont appliqués à effacer les traces de la culture allemande, ce qui apparaît clairement dans le domaine de l'enseignement. Les missions chrétiennes allemandes, chargées de l'enseignement, ont dû quitter le territoire ${ }^{21}$. L'enseignement de la langue française, qui venait remplacer l'allemand et parfois l'anglais, était considéré comme très important. Les autorités ont pu voir le remplacement de la langue allemande par la langue

16 Ibid., p. 3.

17 Ibid., p. 20.

18 MERLIN, Rapport du 06.10.1915, FR ANOM 2300 COL 21. Sur les théories raciales pendant la colonisation allemande, voir Michael SCHUBERT, Der schwarze Fremde. Das Bild des Schwarzafrikaners in der parlamentarischen und publizistischen Kolonialdiskussion in Deutschland von den 1870ern bis in die 1930er Jahre, Diss., Osnabrück, Franz Steiner Verlag Stuttgart, 2001 ; sur les liens entre colonisation et pouvoir, voir Trutz von TROTHA, Koloniale Herrschaft. Zur soziologischen Theorie der Staatsentstehung am Beispiel des "Schutzgebietes Togo”, Tübingen, J. C. B. Mohr, 1994 ; Jürgen ZIMMERER, « Deutsche Herrschaft über Afrikaner: staatlicher Machtanspruch und Wirklichkeit im kolonialen Namibia », EuropaÜbersee, vol. X, Hamburg, LIT, 2001.

${ }^{19}$ MERLin, Rapport du 06.10.1915, FR ANOM 2300 COL 21, p. 9.

${ }^{20}$ FR ANOM 2300 COL 21 et 29.

21 Ulrich van der HEYDEN, Jürgen BECHER (dir.), Mission und Gewalt : der Umgang christlicher Missionen mit Gewalt und die Ausbreitung des Christentums in Afrika und Asien in der Zeit von 1792 bis 1918, Stuttgart, F. Steiner, 2000. 
française comme un premier pas vers l'effacement progressif de la culture allemande au profit de la française. Il importait aussi que les Africains se sentent Français, qu'ils aiment la France et la domination française plus que l'allemande. Ces considérations ressortent des rapports que les nouveaux administrateurs réalisaient, relatant en détail les manifestations francophiles en Afrique ainsi que tous les témoignages faisant état d'une souffrance infligée par les Allemands. La question n'est ici pas de savoir si ces témoignages sont véridiques et justifiés ; toutefois les Français s'intéressaient de près au moindre sentiment antiallemand.

Pour autant, il serait faux de dire que les Français ne possédaient aucune information sur l'organisation allemande : il est par exemple certain qu'ils connaissaient très bien les budgets et le montant des impôts du gouvernorat précédent ${ }^{22}$. En effet, l'une des premières mesures françaises au Cameroun a été d'introduire un impôt calqué précisément sur celui prélevé précédemment par les Allemands. En revanche, selon les dires des administrateurs français, la documentation médicale ainsi que les archives judiciaires avaient disparu ${ }^{23}$. Or, la convention de la seconde Conférence internationale de la Paix du 18 octobre 1907 précisait qu'en cas de changement étatique, la nouvelle puissance devait respecter et appliquer le système de droit de l'État partant :

L'autorité du pouvoir légal ayant passé de fait entre les mains de l'occupant, celui-ci prendra toutes les mesures qui dépendent de lui en vue de rétablir et d'assurer l'ordre et la vie publics, en respectant, sauf empêchement absolu, les lois en vigueur dans le pays. ${ }^{24}$

Ainsi, le but déclaré était d'éviter que les changements d'États produisent une trop grande confusion parmi les populations locales, surtout dans les colonies ${ }^{25}$. Au Cameroun, faute de documentation écrite ou orale sur le fonctionnement de l'administration judiciaire allemande, les Français ont affirmé se trouver face à « un cas d'empêchement absolu » et ont appliqué les lois en vigueur dans la colonie voisine de l'Afrique Équatoriale française :

Les représentants de l'autorité allemande ayant été faits prisonniers ou ayant disparu; (...) les archives ayant été emportées ou détruites; (...) aucun organe d'administration judiciaire n'ayant été maintenu par l'ennemi au moment où il s'est retiré, il convient de remédier à "l'empêchement absolu" de respecter "les

\footnotetext{
${ }^{22}$ Martial Merlin, Exposé Général de la situation dans les Territoires Occupés de l'Ancien Cameroun, FR ANOM 61 COL 1050, p. 47-57 ; et B Arch R1001/3930, Hamburg 22.07.1917, Wirtschaftsdienst Nr.25.

${ }^{23}$ FRANCESCHI, Le mandat français, p. 58, 70, 237-240 ; FR ANOM. 2300 COL 29, Dossier 254 ; MERLIN, Rapport du 06.10.1915, FR ANOM 2300 COL 21, p. 2-9.

${ }_{24}$ Convention de la Haye du 18 octobre 1907, article 43, cité d'après : M. Weiss, Paris 09.09.1915, Le ministère des colonies à Monsieur le Gouverneur général de 1'Afrique Equatoriale Française, FR ANOM 2300 COL 21, Dossier 188.

${ }_{25}$ FR ANOM 2300 COL 29, Dossier 250, « La Législation et les biens ».
} 
lois en vigueur dans les pays" et de répondre, par des mesures de fait, aux nécessités que commande la situation ${ }^{26}$.

Ce tableau est toutefois à nuancer : 1'historien peut aujourd'hui consulter des documents sur la domination allemande à Berlin et à Yaoundé2 ${ }^{27}$, au Cameroun, bien que cette documentation soit nettement moins importante que par exemple celle des archives nationales de Windhoek, en Namibie, où quasiment rien n'a disparu. Nous relevons ici une contradiction qui soulève des questions : les administrateurs français du Condominium ont affirmé que toute la documentation sur les services juridiques allemands avait disparu et qu'ils étaient par conséquent dans l'incapacité d'appliquer les lois allemandes. Pourtant, aujourd'hui encore, à Yaoundé, nous pouvons trouver un nombre certain de documents juridiques allemands. L'historien, à défaut de résoudre cette contradiction, peut proposer des hypothèses : l'absence partielle d'archives impliquait-elle nécessairement l'incapacité des occupants français à étudier les lois indigènes allemandes ? Une partie des archives juridiques aurait-elle été rapatriée plus tard, lorsque la nouvelle administration avait déjà instauré le régime juridique français? Ou alors les administrateurs étaient-ils entrés en possession d'une documentation allemande, passant ce fait sous silence dans le but d'appliquer préférentiellement leur propre système juridique? Deux points peuvent ici être précisés : d'une part, les archives juridiques du gouvernorat allemand qui n'avaient pas été détruites devaient toujours se trouver à Bouéa, en zone anglaise, difficiles d'accès pour les administrateurs français ${ }^{28}$. Par ailleurs, si le gouvernorat français cherchait en vain des lois pénales pour indigènes, c'est surtout parce qu'il n'y en avait quasiment pas. Les colonisateurs allemands n'avaient jamais établi un code pénal détaillé pour leurs sujets africains $^{29}$ et il n'existait que quelques actes réglementaires. En général, les administrateurs locaux allemands, qui pour majorité d'entre eux n'avaient pas reçu de formation juridique, décidaient seuls des sanctions pour les Africains.

Aux difficultés françaises de gérer le nouveau territoire se sont ajoutées les rivalités avec le colonisateur britannique, mieux organisé et mieux équipé. Or, il était important pour la France d'être présente sur ce territoire conquis,

\footnotetext{
${ }^{26}$ Cette mesure concernait toutefois uniquement les territoires qui avaient fait partie de 1'Afrique Équatoriale Française avant d'être cédés à l'Allemagne en novembre 1911, devenant dès lors le « Nouveau Cameroun ", FR ANOM 2300 COL 21, Dossier 192, «Instructions du 23 juin 1916 relatives à l'organisation politique et administrative des Territoires du Cameroun et ayant fait partie de 1'Afrique Équatoriale Française antérieurement à l'accord du 4 novembre $1911 »$.

${ }^{27}$ BArch R175 F, Verwaltung des deutschen Schutzgebietes Kamerun, bearbeitet von Peter GEISSLER, Koblenz 1994, p. II.

${ }^{28}$ FR ANOM 2300 COL 29, dossier 248, 09.02.1918, objet : Guerre franco-allemande. Préméditation d'une attaque allemande des forces anglaises par Ossidinge et Dschang.

${ }^{29}$ Ulrike SCHAPER, Koloniale Verhandlungen. Gerichtsbarkeit, Verwaltung und Herrschaft in Kamerun 1884-1916, Frankfurt/M. / New York, Campus, 2012 ; Isabell SCHEELE, «Le régime pénitentiaire dans le Sud-Ouest allemand africain », mémoire de master 2 non publié, ENS Lyon / Paris 4, 2010.
} 
par son administration, ses infrastructures et ses institutions, ce qui est résumé brutalement par cette citation d'un administrateur : «C'est avec les fusils que l'on chasse l'ennemi ; c'est par les services que l'on s'implante dans le pays $^{30}$. » Cette phrase exprime la volonté de s'implanter dans ce territoire, de se faire accepter par la population et de montrer sa présence afin d'illustrer la bonne gestion du territoire. Un objectif d'autant plus important pour la puissance coloniale que la question de l'appartenance du Cameroun n'était pas résolue. Ce territoire allait-il, après la guerre, être rendu à l'Allemagne ou bien être attribué à l'Angleterre ou à la France? Ainsi, la France entendait ne pas être évincée au moment stratégique du partage des anciennes colonies allemandes. Pour ce faire, il lui fallait, autant que possible, instaurer une administration exemplaire, argument solide dans les négociations à venir.

\section{Une guerre de propagande et une guerre des mémoires (1914-1919)?}

Notre sujet aborde encore d'autres aspects intéressants, qui sont liés à deux nouveautés en particulier : d'une part, l'essor de la propagande de guerre, qui prend des dimensions jusqu'alors inconnues, et d'autre part les deux conventions des Conférences Internationales de la Paix (1899 et 1907) qui ont fixé de manière précise le droit de la guerre et les crimes qui lui sont liés. Parmi ces crimes de guerre figurent : l'assassinat de médecins de la Croix-Rouge, l'exécution de prisonniers de guerre, le pillage, le vol, la destruction de biens et l'utilisation de balles dum-dum ${ }^{31}$, considérées comme excessivement cruelles $^{32}$. Or, les nombreux excès des guerres en Afrique ont fait que toutes les troupes, qu'elles soient allemandes, françaises, britanniques, belges ou africaines, ont commis de tels crimes de guerre ${ }^{33}$. Les différents gouvernements ont systématiquement nié les infractions commises par leurs propres troupes ${ }^{34}$, et lorsqu'ils en ont reconnu certaines, ils en ont rejeté entièrement la faute sur la prétendue "bestialité noire ${ }^{35}$ ». Une « bestialité » qui n'aurait prétendument pas pu être enrayée en raison du manque de personnel blanc. En revanche, ils ont accusé les troupes ennemies et ont détaillé tous leurs méfaits. Des articles accusateurs ont ainsi paru régulièrement dans la presse. Par exemple, le Times britannique a lancé cette querelle, le 16 octobre 1914, avec un article accusant l'Allemagne d'avoir

\footnotetext{
${ }^{30}$ FR ANOM 2300 COL 21, Dossier 192, Douala 17.03.1915, Note.

31 La balle dum dum a une enveloppe en maillechort fendue en croix à l'avant permettant ainsi au noyau de plomb de s'épanouir dans la blessure. Elle a été développée au XIX ${ }^{\mathrm{e}}$ siècle et utilisée par les Anglais en Inde. Lors de la première conférence de La Haye en 1899, elle a été interdite en raison des grandes douleurs qu'elle cause.

${ }^{32}$ SCHULTE-VARENDORFF, Krieg in Kamerun, p. 65.

${ }^{33}$ Ibid., p. 55-70.

${ }^{34}$ Ibid., p. 64.

${ }^{35}$ Ibid., p. 65.
} 
recruté des centaines de $"$ noirs $^{36}$ » dans ses troupes, ce qui aurait forcé les Anglais à faire de même, c'est-à-dire une action contraire aux conventions internationales. Cet article a fait le tour de l'Europe et a alimenté la propagande anti- allemande ${ }^{37}$. Pour contrer ces allégations, l'Office impérial aux Colonies se proposait de publier des extraits d'archives dans le Norddeutsche Allgemeine Zeitung ${ }^{38}$. Par la même occasion, la presse devait accuser l'Angleterre d'avoir incité des troupes noires à piller et à tuer des civils. L'Office impérial aux Colonies ${ }^{39}$ prévoyait ainsi de publier des témoignages de missionnaires et de civils sur les prétendus pillages massifs de propriétés privées, sur les mauvais traitements dans les camps de prisonniers de guerre ainsi que sur une population africaine que les Britanniques auraient dressée contre les Allemands, donnant soi-disant lieu à une véritable chasse aux Allemands. Il est à noter que, dans le contexte des théories raciales de l'époque, les colons se sont sentis particulièrement offensés d'avoir été fait prisonniers par des Africains ${ }^{40}$.

Le ton excessif et le contexte de la guerre permettent d'affirmer que le but premier de ces publications était de donner une image négative de l'ennemi et de faire croire à l'immoralité de la partie adverse. L'Allemagne et les Alliés se sont mutuellement accusés de crimes de guerre ou d'autres «atrocités ${ }^{41}$ " commises en Afrique. Le gouvernement allemand est même allé jusqu'à demander, mais en vain, la médiation des États-Unis contre cette politique de guerre qui offensait tout « sentiment de la race ${ }^{42} »$. Il s'agit ici d'une véritable guerre de propagande, menée à grands renforts d'extraits d'archives publiés par voie de presse, dont l'objectif était indéniablement de rallier l'opinion nationale et l'opinion internationale contre l'ennemi.

Il n'est pas excessif d'affirmer que ces tentatives se sont soldées par un échec. Le traité de paix signé à Versailles en 1919 en est la preuve : l'Allemagne a été destituée du droit à la colonisation. Et l'un des principaux arguments était d'ordre humanitaire et moral : le gouvernorat colonial aurait été particulièrement brutal dans ses colonies. L'opinion internationale s'était en effet retournée contre l'Allemagne lorsque le gouvernement britannique

\footnotetext{
${ }^{36}$ BArch R 1001/7015, Kriegssachen 1914-1915, A. W. SCHREIBER, Stellvertretender Vorsitzender der Gesellschaft für Eingeborenen-Schutz an das RKA, Berlin 05.12.1914, Betr. : Beteiligung farbiger Truppen am Kolonial-Kriege.

37 Ibid. Il a été par exemple publié dans le journal suisse La semaine religieuse.

${ }^{38}$ Ibid.

39 BArch R1001/3987, Deutscher Evangelischer Missionsausschluss an das RKA, Herrnhut, 29.01.1916, Betr. : Blaubuch der englischen Regierung vom November 1915.

${ }^{40}$ ReICHS-KolOnialamt, Die Kolonialdeutschen aus Kamerun und Togo in französischer Gefangenschaft, Berlin, Reichsdruckerei, 1917.

${ }^{41}$ MerLin, Rapport du 06.10.1915, FR ANOM 2300 COL 21, p. 19.

42 « Diese Kriegsführung verstößt gegen jedes Rassegefühl [...] », BArch R1001/3987, Auswärtiges Amt an den Herrn Staatssekretär des Reichs-Kolonialamts, Berlin 14.06.15, Abschrift eines am 31.5.15 an die hiesige Botschaft der Vereinigten Staaten von Amerika gerichteten Verbalnote wegen der Kriegsführung der britisch-französischen Truppen in Kamerun.
} 
avait publié un «livre bleu ${ }^{43}$ » sur une politique coloniale qui, selon les auteurs anglais, n'avait été qu'exploitation et barbarie. Le président des ÉtatsUnis, Woodrow Wilson, s'est rangé du côté des Alliés contre l'Allemagne, et les anciennes colonies allemandes ont été transformées en mandats sous tutelle de la Société des Nations. Cette argumentation a été particulièrement douloureuse pour les avocats de la colonisation allemande, qui considéraient qu'une telle entreprise était un reflet du stade de civilisation d'une nation. Affirmer que l'Allemagne était incapable d'administrer correctement ses colonies revenait à lui renvoyer à la figure qu'elle était moins civilisée que les autres nations européennes. En Allemagne est alors née la légende du " mensonge sur la faute coloniale ${ }^{44}$ », point central d'un révisionnisme qui a eu une influence non négligeable sur la politique allemande jusqu'en 1945. Là encore se sont affrontées différentes campagnes de propagande, allemande, française et britannique. Pour ne citer qu'un seul exemple, le 5 octobre 1918, la société coloniale de Brême a suggéré au secrétaire d'État Wilhelm Solf de répondre aux «diffamations ${ }^{45}$ " anglaises. Le moyen envisagé a été la publication d'extraits d'un projet de loi datant de 1913, destiné à mieux protéger les travailleurs "indigènes ». La société de Brème plaidait sa cause en soulignant que le souci humaniste était plus important chez les colonisateurs allemands, les lois britanniques ne garantissant pas semblable protection.

D'autre part, de nombreuses publications ont fait naitre le mythe des fidèles Africains qui, par centaines de milliers, auraient suivi les colonisateurs allemands en territoire espagnol, et qui n'auraient souhaité qu'une chose, leur retour au Cameroun. Notons que les archives françaises affirment exactement le contraire : la quasi-totalité des chefs de tribu ont rédigé et signé une déclaration dans laquelle ils ont refusé le retour des Allemands et affirmé vouloir être gouvernés par la France ${ }^{46}$. Ces déclarations et témoignages étaient d'une importance primordiale pour le colonisateur français, qui pouvait s'en servir pour appuyer sa demande de voir rattacher ces territoires à son empire. On est donc fondé à se demander si ces déclarations, ces manifestations et ces témoignages étaient entièrement spontanés ou s'ils ont

\footnotetext{
${ }^{43}$ Jeremy SilVESTER, Jan-Bart GeWALD, Words cannot be found. German Colonial Rule in Namibia. An Annotated Reprint of the 1918 Blue Book, Sources for African History, Leiden / Boston, Brill, 2003.

${ }_{44}$ "Kolonialschuldlüge ». Sur le révisionnisme colonial, voir par exemple Christoph RogOWSKI, "Heraus mit unseren Kolonien!” Der Kolonialrevisionismus der Weimarer Republik und die "Hamburger Kolonialwoche" von 1926 », in Birthe KUnDRUS (dir.), Phantasiereiche. Zur Kulturgeschichte des deutschen Kolonialismus, Frankfurt/M., Campus, 2003, p. 243-262.

45 « Verleumdungen », BArch R1001/3233, Bremer Kolonial-Handelsgesellschaft an den Staatssekretär des RKA Herrn Dr.Solf, Bremen, 05.10.1918, p. 2-3.

${ }^{46}$ FR ANOM 61 COL 1050, Dossier non numeroté « Manifestations de loyalisme », et FR ANOM 2300 COL 21, Dossiers 188 et 189. Sur la volonté française de mettre le territoire camerounais en valeur, voir Walter HERTH, "Mise en valeur" und Weltwirtschaftskrise. Koloniale Entwicklungspolitik in Kamerun unter französischer Herrschaft 1916-1938, Diss., Universität Zürich, Zentralstelle der Studentenschaft, 1988.
} 
été partiellement provoqués à l'aide de divers moyens par les autorités françaises.

\section{Conclusion}

La destruction de documents sous le secret militaire semble avoir été une mesure de contre-espionnage couramment appliquée, et les archives ne sont certainement pas ce qui a le plus occupé les gouvernorats en temps de guerre. Toutefois, lorsque les gouvernements européens ont publié des extraits d'archives pour servir leur propagande, ils pratiquent peu ou prou une guerre spécifique des archives. Les extraits d'archives sont ainsi devenus une arme utilisée par la presse et par les organes de propagande des gouvernements allemand, français et britannique. Ils étaient alors considérés comme des preuves solides, ce qui n'excluait nullement leur manipulation. Les organes de propagande se sont servis de ces preuves prétendues incontestables afin de clamer publiquement les torts et méfaits de l'ennemi, de l'attaquer sur un plan moral, dans le but de rallier l'opinion publique internationale. Si cet aspect prend une ampleur considérable au cours de la guerre et de l'élaboration du Traité de paix, c'est en raison du rôle inédit des conventions internationales sur le droit de la guerre. Ainsi, la question juridique et morale des crimes de guerre a eu des conséquences assez surprenantes et peu connues.

D'autre part, la disparition des archives coloniales allemandes rend parfois la recherche impossible. D'éminents chercheurs, dont l'ethnologue Claude $\operatorname{Tardits}^{47}$, en France, et l'historien Peter Sebald, en Allemagne, ont recherché en vain des archives coloniales allemandes. Peter Sebald espérait retrouver une cinquantaine de dossiers, dont il pensait avoir réussi à retracer l'itinéraire avec succès : ces dossiers auraient été transférés de Paris vers Berlin en 1940, puis vers Moscou en 1945 et de nouveau vers Paris en 1994. Voici quelques précisions : en 1940, plus de mille caisses d'archives ont été transférées depuis Paris occupée vers Berlin. C'était une entreprise assez mal organisée, le premier arrivé pouvant souvent prendre des documents sans même établir une liste des archives emportées. Il est sûr que parmi cette énorme masse de documents se trouvaient au moins deux dossiers allemands sur le Cameroun, datés de $1912^{48}$. Lorsque les troupes russes ont conquis Berlin en 1945, elles ont emporté ce fonds d'archives français ainsi qu'un volume inconnu de documents historiques allemands. Ces fonds ont été entreposés dans un centre spécial près de Moscou, dont l'existence même a été tenue secrète jusqu'en 1990. À la suite d'un accord franco-russe de 1992, deux tiers des fonds français ont été remis à la France en 1994, où ils ont été entreposés dans les

\footnotetext{
47 Claude TARDITS, Contribution à l'étude des populations Bamiléké de l'ouest Cameroun, Paris, Éd. Berger-Levrault, 1960, p. 114.

${ }^{48}$ ANF 20010217/5, Dossier 316, Paris, 15.09.1941, Sendung 60, Betr. : Aufstellung von Schriften und Aktenmaterial, das von Herrn Abteilungsleiter Rothhaupt aus dem französischen Kolonialministerium entnommen wurde zum Versand an das Kolonialpolitische Amt Berlin.
} 
Archives nationales contemporaines à Fontainebleau. En revanche, en 1997, le gouvernement russe a refusé de remettre à l'Allemagne ses fonds historiques. Il n'est donc pas impossible que des archives coloniales allemandes se trouvent toujours dans ce centre spécial en Russie. Peter Sebald et l'auteure de l'article avaient espéré trouver des documents allemands à Fontainebleau, qui auraient été entreposés là par erreur, mais cette hypothèse est impossible à vérifier (en effet, une partie importante du fonds de Moscou se trouve dans un bâtiment contaminé par l'amiante ${ }^{49}$ ) et demeure peu probable.

\footnotetext{
Damien RicharD, «Le "fonds de Moscou" aux Archives nationales - site de Fontainebleau ", Service des publics, Archives Nationales, mise en ligne en juillet 2010, http://www.archivesnationales.culture.gouv.fr/chan/chan/pdf/103-fonds-moscou.pdf, consulté le 09.12.2013.
} 